\title{
Efficacy of tigecycline monotherapy versus combination therapy with other antimicrobials against carbapenem-resistant Acinetobacter baumannii sequence type 2 in Heilongjiang Province
}

\author{
Jiaying Li ${ }^{1 \#}$, Yanjun Fu ${ }^{1 \#}$, Jisheng Zhang ${ }^{2}$, Ying Wang ${ }^{3}$, Yongxin Zhao ${ }^{1}$, Xuecai Fan ${ }^{4}$, Lan Yu ${ }^{1}$, \\ Yong Wang ${ }^{3}$, Xiaoli Zhang ${ }^{2}$, Chunjiang $\mathrm{Li}^{1}$ \\ ${ }^{1}$ Department of Pathogenic Biology, Jiamusi University School of Basic Medicine, Jiamusi 154007, China; ${ }^{2}$ Department of Microbiology, Yongchuan \\ Hospital of Chongqing Medical University, Chongqing 402160, China; ${ }^{3}$ Department of Microbiology, The First Affiliated Hospital of Jiamusi \\ University, Jiamusi 154002, China; ${ }^{4}$ Department of Microbiology, The Second Affiliated Hospital of Jiamusi University, Jiamusi 154002, China \\ Contributions: (I) Conception and design: X Zhang, J Li, Y Fu; (II) Administrative support: X Zhang, C Li; (III) Provision of study materials or \\ patients: J Zhang, Y Wang; (IV) Collection and assembly of data: X Fan, Y Zhao, L Yu, Y Wang; (V) Data analysis and interpretation: C Li, J Li, J \\ Zhang; (VI) Manuscript writing: All authors; (VII) Final approval of manuscript: All authors. \\ \#These authors contributed equally to this work. \\ Correspondence to: Xiaoli Zhang. Department of Microbiology, Yongchuan Hospital of Chongqing Medical University, Chongqing 402160, China. \\ Email: jmszx1123@163.com; Chunjiang Li. Department of Pathogenic Biology, Jiamusi University School of Basic Medicine, Jiamusi 154007, China. \\ Email: 3355806796@qq.com.
}

Background: Selecting alternative antibiotic combinations as treatment options may help successfully manage carbapenem-resistant Acinetobacter baumannii (CRAB). This study aimed to determine the synergistic effects of tigecycline (TIG) monotherapy versus combination therapy with other antimicrobials against CRAB.

Methods: After performing biochemical identification assays, we detected oxacillin-hydrolyzing (OXA)-type carbapenemase genes in $35 \mathrm{CRAB}$ isolates. The minimum inhibitory concentrations (MICs) and interactions of the test drugs were determined using the checkerboard assay with TIG, colistin (CST) and meropenem (MEM). Static time-kill assays were conducted to validate the synergistic effects of the most efficacious combination.

Results: The chromosomal gene, bla $a_{O X A-51-l i k e}$, was tested among all isolates, bla $a_{O X A-23-l i k e}$ and bla $a_{\text {OXA-24-like }}$ were present in $91.4 \%$ and $25.7 \%$, respectively. In the checkerboard assay, the combination of TIG and MEM displayed the highest rate of synergy (30.5\%) against the 35 isolates. In contrast, the TIGCST combination showed a higher indifference interaction rate $(36.1 \%)$ than that of the TIG-MEM (16.7\%) combination. Antagonism appeared in one isolate for the TIG-CST combinations. The static time-kill assays confirmed the superior synergistic effect of CST against the CRAB isolates.

Conclusions: TIG combined with CST exhibited early synergistic activity that was not sustained beyond $12 \mathrm{~h}$. TIG combination therapy can only be recommended when other optimized therapeutics are unavailable.

Keywords: Acinetobacter baumannii; tigecycline (TIG); in vitro; colistin (CST)

Submitted Sep 09, 2019. Accepted for publication Nov 05, 2019.

doi: 10.21037/apm.2019.11.06

View this article at: http://dx.doi.org/10.21037/apm.2019.11.06 


\section{Introduction}

In recent decades, carbapenem-resistant Acinetobacter baumannii (CRAB) has become one of the most important causative agents associated with hospital-acquired infections worldwide (1). Developing new antimicrobial agents and therapeutic strategies has been a major challenge in the field of infectious diseases. Various effective antibacterial drugs are available but often exert adverse effects on patients. Currently, tigecycline (TIG) is the best option for treating and controlling CRAB-related infections (2).

TIG is the irreplaceable member of the glycylcycline class of antimicrobial agents, the Food and Drug Administration (FDA) has been approved it to treat complicated skinstructure infections, intraabdominal infections and community-acquired pneumonia (3). TIG inhibits bacterial protein synthesis by acting on the $30 \mathrm{~S}$ ribosomal subunit and prevents amino acids from incorporating and elongating peptide chains (4). Randomized trials have shown that use TIG alone may increase the risk of death, which further suggests that TIG is best used for combination therapy (5). However, because most of these researches concentrated on multisite infections with multiple pathogens, establishing the effect of TIG as a single agent against CRAB is difficult because of the many confounding factors. Thus, whether TIG is effective in treating CRAB-related infections remains unclear.

Therapeutic choices for treating Acinetobacter spp. are usually limited to polymyxins and TIG. Empiric antibiotic therapy for $A$. baumannii should be chosen based on local susceptibility patterns (6). The treatment should contain a broad-spectrum cephalosporin combined with a carbapenem. Fluoroquinolones, aminoglycosides, or colistin (CST) are second-line alternatives. Selecting appropriate alternative treatments with antibiotic combinations may help successfully manage CRAB infections.

Because TIG-based combination therapy is valuable in treating TIG-resistant Acinetobacter baumannii, we conducted this study on CRAB to assess the effectiveness of TIG alone or combined with other drugs in treating CRAB and to identify the forecasts of therapy success.

\section{Methods}

\section{Collection of patients' clinical data and bacterial strains}

Thirty-five CRAB clinical isolates were obtained from the First Affiliated Hospital of Jiamusi University between September 2013 and June 2015. A. baumannii was initially identified by the Vitek 2 system (bioMérieux, Marcyl'Etoile, France). Clinical characteristics of the CRABinfected patients were collected, including age, sex, sample type, admission ward, prior antimicrobial use, recovery situation, and outcome. Of the 35 strains, 29 were isolated from sputum, three from cerebrospinal fluid, one from pus, one from blood, and one from wound secretions. $A$. baumannii ATCC 19606 and Escherichia coli ATCC 25922 were used as reference strains. The isolates were put away $-80{ }^{\circ} \mathrm{C}$ until use and subcultured on Columbia agar for in vitro testing.

\section{Multilocus sequence typing (MLST) and detection of oxacillin-hydrolyzing (OXA)-type carbapenemase genes}

$\beta$-lactamase-associated genes were amplified via PCR using primers as described previously (7). Table 1 lists several primer sets used to amplify $\beta$-lactamases of Ambler classes A, $\mathrm{B}, \mathrm{C}$, and D (bla OXA-23-like, $b l a_{O X A-24-l i k e}$, and bla OXA-51-like; http:// pubmlst.org/abaumannii/) (8).

To estimate the relationship between MLST type and the isolates, isolates were arranged according to the time. Put the same isolates via PCR detected the genes encoding carbapenemases. Adopting BLAST (http://blast.ncbi.nlm. nih.gov/) (9) to compared nucleotide sequences gained from PCR sequencing. By PCR and sequencing analysis of seven housekeeping genes to performed MLST. Then compared the nucleotide sequences obtained sequencing with sequences preexisting in the MLST databases to distribute the allelic numbers and sequence types (STs).

\section{Checkerboard assay for synergy testing}

The minimum inhibitory concentrations (MICs) for TIG, CST and meropenem (MEM) were defined by agar dilution in accordance with CLSI recommendations. CST used in the research was purchased from Sigma-Aldrich (St. Louis, MO, USA). TIG was purchased from Wyeth Pharmaceutical Co. Ltd., and MEM was from Haibin Pharmaceutical Co., Shenzhen, China. According to the CLSI breakpoint criteria, the MIC results were explained.

In the guidelines, no breakpoints for TIG are used. So adopt the criteria of the United States FDA for Enterobacteriaceae for TIG (susceptibility, $\leq 2 \mu \mathrm{g} / \mathrm{mL}$; resistance, $\geq 8 \mu \mathrm{g} / \mathrm{mL}$ ) (10). Synergistic testing was performed in 96 -well microdilution plates (8). The synergistic effect was assessed by the fractional inhibitory concentration index (FICI), which was calculated as follows: 


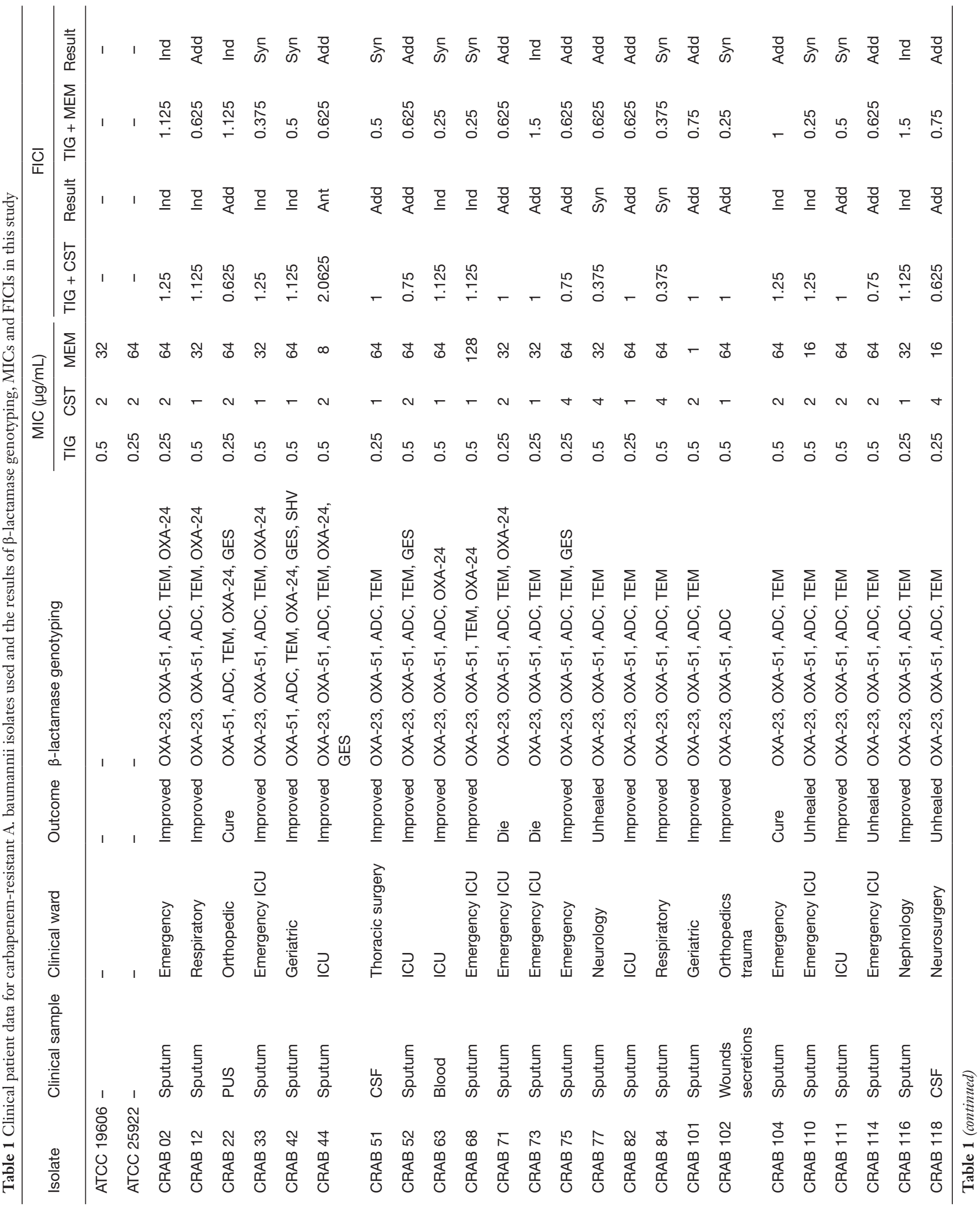




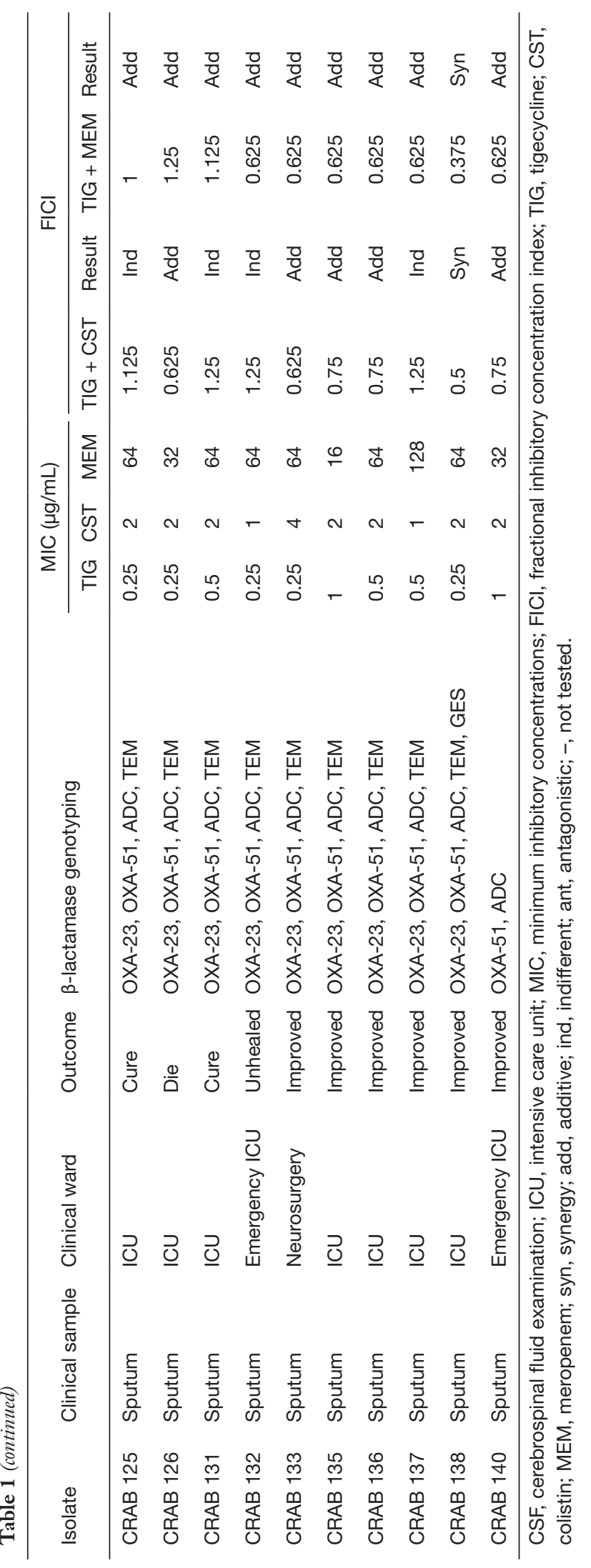

$$
\mathrm{FICI}=\mathrm{MIC}_{(\mathrm{a} \text { in combination })} / \mathrm{MIC}_{(\mathrm{a})}+\mathrm{MIC}_{(\mathrm{b} \text { in combination })} / \mathrm{MIC}_{(\mathrm{b})} \text {. }
$$

The FICI was interpreted as follows: synergistic: FICI is smaller 0.5; antagonistic: FICI greater than 4.0; and indifferent: $0.5<$ FICI $<4.0$. Synergy was also defined as an SBPI $>2$ as calculated using the checkerboard assay (11).

\section{Time-kill assays}

By the CLSI guidelines [2015], the time-kill assays were in progress in triplicate for four representative isolates. In short, concentrations of $1 \times$ and $0.5 \times$ MIC were prepared in Mueller-Hinton broth for TIG alone and combined with CST or MEM. The bacterial suspension was incubated at $37^{\circ} \mathrm{C}$ with swinging at $160 \mathrm{rpm}$ for $24 \mathrm{~h}$. We also had an antibiotic-free growth control. The predetermined timepoints were $0,4,8,12$ and $24 \mathrm{~h}, 100-\mu \mathrm{L}$ samples were obtained, then diluted, and spread on Mueller-Hinton agar plates to observe the colony-forming units (CFU) per mL. Establish a time-kill curve function relationship, The results are expressed as the difference in $\log 10$ at each time point at 0 to $24 \mathrm{~h}$. Comparing the antibiotics in combination to the most active drug at the stationary time-point, decrease of $\geq 3 \log 10$ indicated bactericidal, decrease of $\geq 2 \log 10$ was synergistic effects, while an increase of $>2 \log 10$ was antagonism (12).

\section{Statement of ethics approval}

The study protocol was approved by the Ethics Committee of Jiamusi University Clinical Medical College for research. The committee's reference number is 059 . Individual informed consent was waived by the ethics committee listed above because this study used currently existing sample collected during the course of routine medical care and did not pose any additional risks to the patients.

\section{Results}

\section{Collection of patients' clinical data and bacterial strains}

Table 1 presents the patients' clinical data. Among these isolates, $45.7 \%$ were healed and they were from the intensive care unit, of whom, $25.7 \%$ were women with a median age of 53 years. The male patients had a median age of 64 years. Of the isolates, $80 \%$ were collected from the respiratory tract, with the remainder having been obtained from other sources. All 35 CRAB isolates were susceptible to TIG, while $85.7 \%$ and $3.0 \%$ were susceptible to CST 
Table 2 The mic $_{50}$ and mic $_{90}$ values and susceptibility

\begin{tabular}{lcccc}
\hline \multirow{2}{*}{ Antibiotics } & \multicolumn{3}{c}{ MIC result $(\mu \mathrm{g} / \mathrm{mL})$} & Susceptibility (\%) \\
\cline { 2 - 4 } & $50 \%$ & $90 \%$ & Range & \\
\hline Tigecycline & 0.5 & 0.5 & $0.25-1$ & 100 \\
Colistin & 2 & 4 & $1-4$ & 85.7 \\
Meropenem & 64 & 64 & $1-128$ & 3.0 \\
\hline
\end{tabular}

MIC, minimum inhibitory concentrations.

Table 3 Results of the checkerboard synergy test of combination of tigecycline with two different antibiotics against carbapenem-resistant Acinetobacter baumannii isolated

\begin{tabular}{lcccc}
\hline \multirow{2}{*}{ Combination } & \multicolumn{4}{c}{ Result } \\
\cline { 2 - 5 } & Synergy & Additive & indifferent & Antagonist \\
\hline Tigecycline + Colistin & $8.5 \%$ & $51.5 \%$ & $37.2 \%$ & $2.8 \%$ \\
Tigecycline + & $28.6 \%$ & $60.0 \%$ & $11.4 \%$ & $0.0 \%$ \\
Meropenem & & & & \\
\hline
\end{tabular}

and MEM, respectively. Table 2 presents the $\mathrm{MIC}_{50}$ and $\mathrm{MIC}_{90}$ values and susceptibility results.

\section{MLST and OXA carbapenemase gene detection}

MLST revealed that all CRAB isolates were ST2. The PCR results showed that several CRAB isolates carried multiple resistance genes. The PCR assay for common carbapenemase genes showed that $b l a_{O X A-23-l i k e}$ and $b l a_{O X A-24-l i k e}$ were present in $91.4 \%$ and $25.7 \%$ of the isolates, respectively, and seven isolates harbored both $b l a_{O X A-23-l i k e}$ and $b l a_{\text {OXA-24-like }}$ genes. The chromosomal bla OXA-51-like gene was detected in all tested isolates (Table 1).

\section{Checkerboard synergy analysis}

Table 1 shows the checkerboard synergy testing results for the 35 CRAB isolates. The TIG-CST combination showed a synergistic effect against three of the 35 tested isolates $(8.5 \%)$, an additive effect against 18 isolates $(51.5 \%)$, and an indifferent effect against 13 isolates (37.2\%), with FICI values of $0.375-2.0625$. The TIG-MEM combination showed an additive effect against 21 strains $(60.0 \%)$ and an indifferent effect against four strains (11.4\%); 4 of these carried bla $a_{\text {OXA-24-like, }}$ with FICI values of $0.375-1.5$. Table 3 shows the results of the combination checkerboard synergy testing.

\section{Time-kill assays}

For the time-kill studies, four isolates with different carbapenem-resistance mechanisms and belonging to the same STs were exposed to TIG alone or in combination at concentrations of $1 \times$ or $0.5 \times$ the MICs of TIG. At $0.5 \times$ the MICs of TIG, the combination with CST showed both synergy and bactericidal activity in two isolates carrying $b l a_{O X A-23-l i k e}$ and bla $a_{O X A-24-l i k e}(\mathrm{CRAB} 22$ and CRAB52). CRAB42 also carried $b l a_{O X A-24-l i k e}$, which showed antagonistic activity at $24 \mathrm{~h}$. All isolates showed antagonistic activity toward MEM (Figure 1). The combination of $1 \times$ the MIC of TIG with high-concentration CST showed synergy against $75 \%$ of the isolates (Figure 2). Isolates CRAB52 and CRAB125 carried $b l a_{O X A-23-l i k e}$ and exhibited synergy. These results indicate that the combination of TIG and CST is effective against CRAB isolates. Furthermore, the combinations of TIG with high or low concentrations of MEM were antagonistic. Compared with the combination antibiotics, the single drug CST had better antibacterial effects, even at low concentrations $(4 \mu \mathrm{g} / \mathrm{mL})$.

\section{Discussion}

Acinetobacter baumannii is a pathogen in hospitals characterized by inborn and acquired antimicrobial resistance (13). Of main concern is the increased incidence of carbapenem resistance, which has risen markedly in the past decade, and there are few treatment options (14).

Of CRAB isolates, production of acquired carbapenemhydrolyzing class $\mathrm{D} \beta$-lactamases, which can confer carbapenem resistance (15), and overproduction of intrinsic class D $\beta$-lactamase $b l a_{O X A-51-\text { like }}$ family members are the most prevalent mechanisms of carbapenem resistance. The $b l a_{O X A-23-l i k e}, b l a_{O X A-24-l i k e}$ and $b l a_{O X A-58-l i k e}$ genes and the recently discovered $b l a_{O X A-143-l i k e}$ and $b l a_{O X A-253-l i k e}$ genes are globally associated with CRAB emergence (16). Bla OXA23-like is the most prevalent enzyme associated with CRAB infections. Significant associations of the bla $a_{\text {OXA-23-like }}$ gene and carbapenem resistance have been observed, and this phenomenon is consistent with our findings. The chromosomal gene $b l a_{O X A-51-l i k e}$ was detected in all isolates, and $b l a_{O X A-23-l i k e}$ was present in $91.4 \%$ of the isolates.

Although the clinical role of TIG for treating CRAB infections remains controversial because of limited efficacy and increasing resistance, TIG remains a beneficial option in specific situations such as when given as largedose therapy or as part of combination regimens (17). 

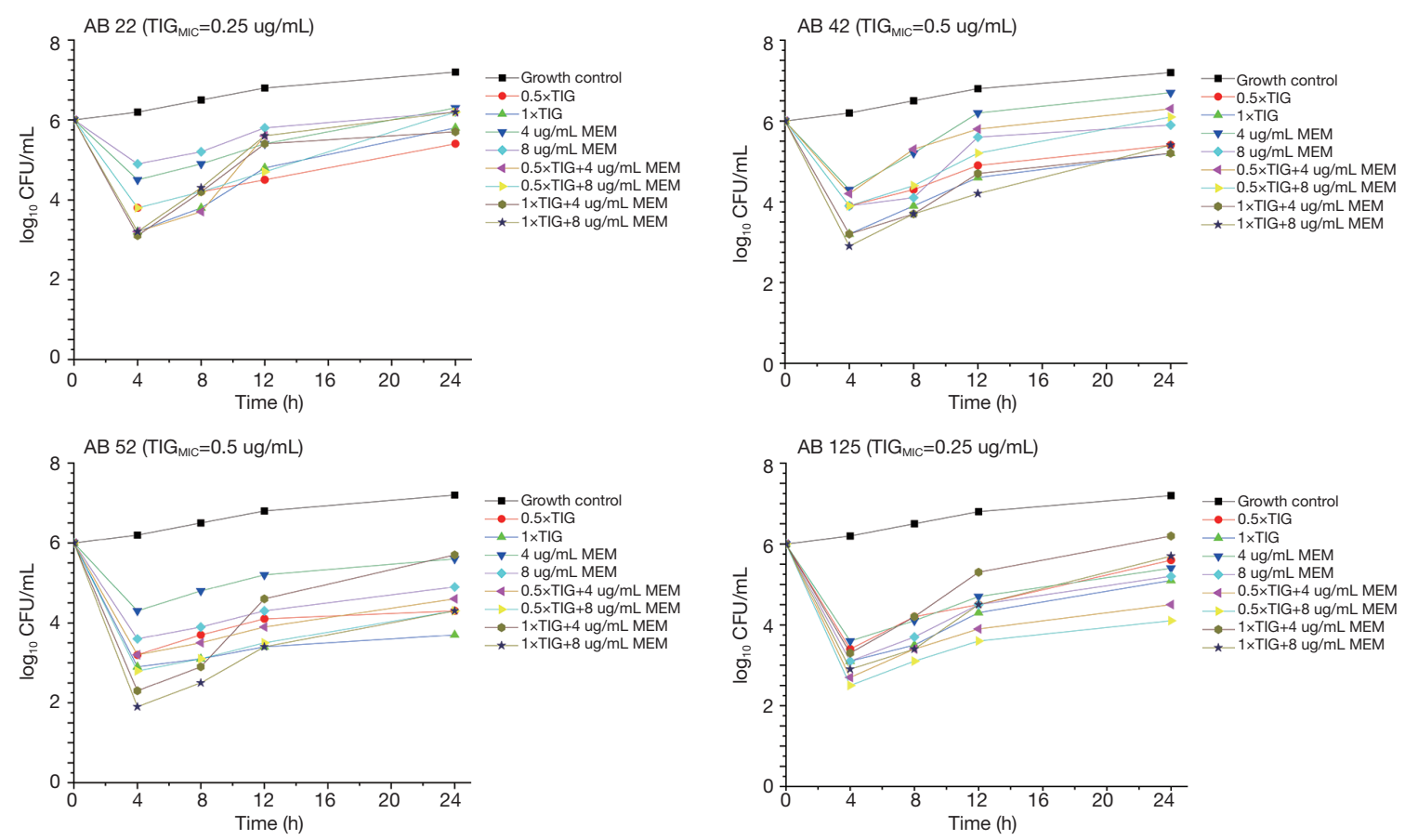

Figure 1 Results of dynamic time-kill experiments with tigecycline alone and in combination with meropenem.
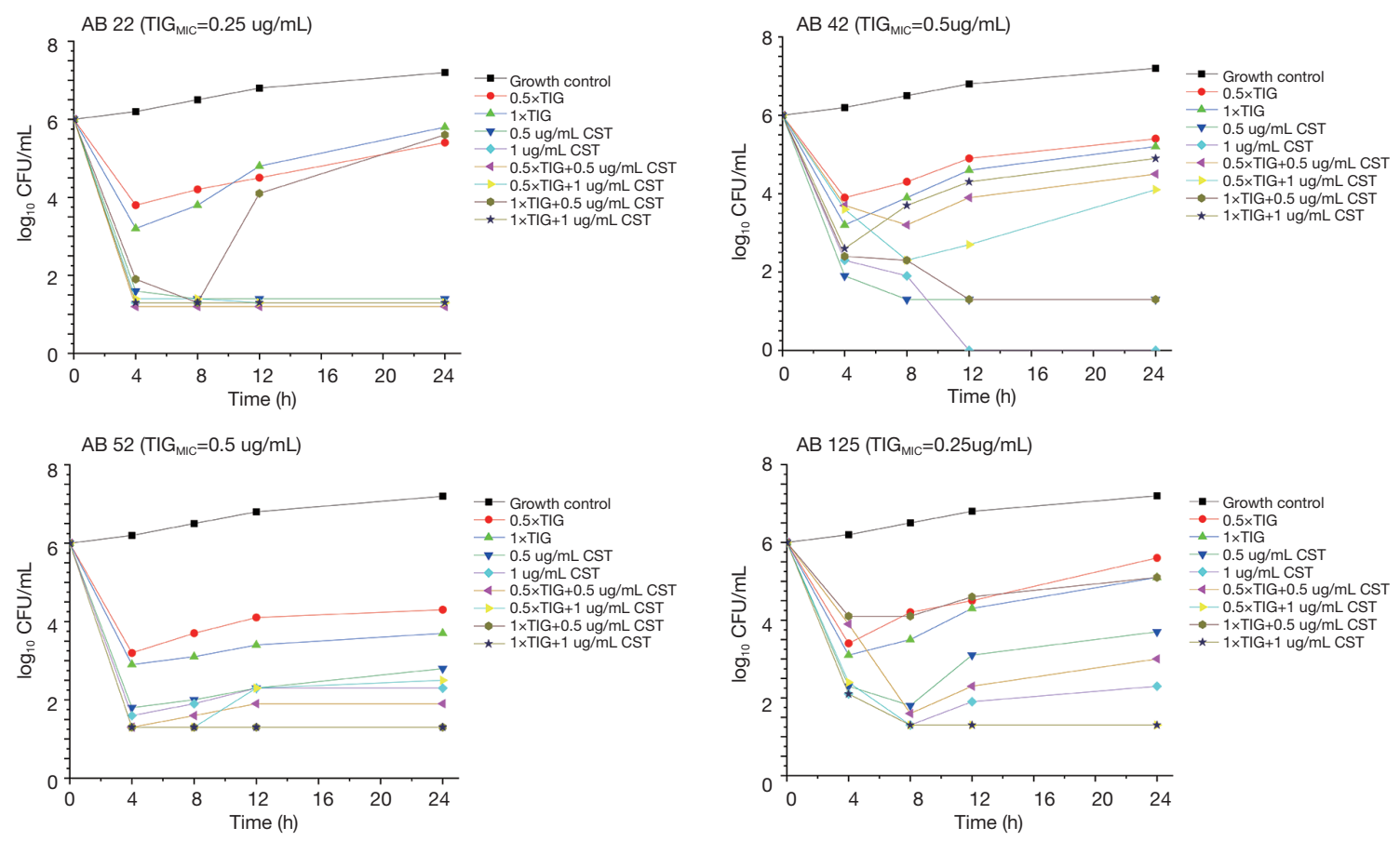

Figure 2 Results of dynamic time-kill experiments with tigecycline alone and in combination with colistin. 
The present study demonstrated a difference in the effectiveness of TIG combined with CST or MEM against CRAB isolates from northeastern China, and all CRAB isolates were susceptible to TIG. However, using TIG as a monotherapy promotes the emergence of heteroresistant $A$. baumannii isolates and the development of TIG resistance among other bacterial species. The MICs of the drugs in the combination treatments were reduced compared with the MIC of each drug alone, thereby reducing the required dose. When TIG was used alone, the TIG concentration was very low. The MIC showed that TIG inhibited $94 \%$ of the bacteria at less than or equal to $0.5 \mu \mathrm{g} / \mathrm{mL}$ based on the criteria of the United States FDA; thus, all CRAB isolates were sensitive. The MIC results showed that all isolates were sensitive to TIG when they carried the $b l a_{O X A-23-\text {-like }}$ or bla $a_{\text {OXA-24-like }}$ genes. In addition, $14.30 \%$ of the CRAB isolates were resistant to CST and carried $b l a_{O X A-23-l i k e}$.

In this study, 32 isolates carried the bla $a_{O X A-23-l i k e}$ gene. The TIG-MEM combination showed a low rate of synergy (in $28.1 \%$ of strains), and $44.4 \%$ of the nine isolates with $b l a_{\text {OXA-24-like }}$ showed synergism. The time-kill study revealed that MEM monotherapy had little discernible effect. Antagonism was dominant in the TIG plus MEM combinations, which may be because the $\beta$-lactam antibiotics act within the periplasmic space and primarily pass though the outer membrane (18). TIG plays a part in the cytoplasm by inhibiting the $30 \mathrm{~S}$ subunit of the ribosome and must through two membranes (19). However, we found that the results for TIG-MEM differed between the checkerboard synergy analysis and the time-kill assays. CRAB42 had a FICI of 0.5 on the checkerboard synergy analysis, representing synergy, but this isolate showed antagonism in the time-kill assays. The difference in this result was due to the time-kill assays; we used clinically achievable concentrations to determine the antibacterial effects. Therefore, the results obtained in our study are more useful for clinical practice. We collected specimens from Heilongjiang Province, which were all ST2 type. These specimens differed from those in Park et al.'s study (20); therefore, the results differed, although all specimens contained bla $a_{O X A-23-\text {-like }}$. Thus, the impact of different MLSTtyping methods on efficacy requires further verification.

Hagihara et al. (21) found that CST was responsible for most of the effect against CRAB isolates tested with CST and TIG. Similar to our observations, TIG monotherapy showed a lack of activity. CST alone achieved more significant bactericidal activity than did TIG, especially the TIG/CST combination. The results from the present study are consistent with the findings of Rao et al. (22), who revealed that CST combined with TIG against $C R A B$ isolates in the time-kill assay resulted in early bactericidal activity within $4 \mathrm{~h}$ at concentrations sustained for $24 \mathrm{~h}$. When the CST concentration reached $1 \mu \mathrm{g} / \mathrm{mL}$ at $24 \mathrm{~h}$, three isolates had synergy due to the increased active uptake of TIG into the cell, resulting in sufficient intracellular concentrations to slow bacterial growth by inhibiting protein synthesis. In addition, CST may increase TIG penetration at the action site by permeabilizing the outer bacterial membrane.

This study had several limitations. First, the bactericidal effects of the combinations were only examined for $24 \mathrm{~h}$. Therefore, whether resistance and regrowth occurred after $24 \mathrm{~h}$ is unknown. In addition, only four CRAB strains were used in the present study; thus, the representativeness of these results must be further verified with more isolates from other species. However, to our knowledge, this is the first report on TIG combination therapy against $A$. baumannii in Hei Longjiang Province.

\section{Conclusions}

TIG combination therapy does not eradicate microbes, but it reduces bacterial loads and adverse drug reactions. CST is currently unavailable on the Chinese market. Therefore, TIG combination therapy may be a reasonable alternative before more optimized treatments become available. However, TIG is a bacteriostatic agent and may not be recommended as a first-line treatment based on current research that does not show a benefit toward the end result. Further studies based on PK/PD parameter combination therapy should be performed to explore the optimal dose, route of administration, and optimal combination of TIG.

\section{Acknowledgments}

Funding: This work was supported by Excellent Team of Young Teachers Foundation of Heilongiiang Province (2018-KYYWF-0916), Heilongjiang Provincial Health and Family Planning Commission on Scientific Research Project (2018-290 and 2018-291), Jiamusi University President Innovation and Entrepreneurship Fund Project (XZYF2018-39) and Research Project of Yongchuan Hospital Affiliated to Chongqing Medical University (YJYJ201902). 


\section{Footnote}

Conflicts of Interest: The authors have no conflicts of interest to declare.

Ethical Statement: The authors are accountable for all aspects of the work in ensuring that questions related to the accuracy or integrity of any part of the work are appropriately investigated and resolved. The study protocol was approved by the Ethics Committee of Jiamusi University Clinical Medical College for research. The committee's reference number is 059 . Individual informed consent was waived by the ethics committee listed above because this study used currently existing sample collected during the course of routine medical care and did not pose any additional risks to the patients.

\section{References}

1. Zilberberg MD, Kollef MH, Shorr AF. Secular trends in Acinetobacter baumannii resistance in respiratory and blood stream specimens in the United States, 2003 to 2012: A survey study. J Hosp Med 2016;11:21-6.

2. Berditsch $M$, Jäger $T$, Strempel $N$, et al. Synergistic effect of membrane-active peptides polymyxin $\mathrm{B}$ and gramicidin $\mathrm{S}$ on multidrug resisitant strains and biofilms of pseudomonas aeruginosa. Antimicrob Agents Chemother 2015;59:5288.

3. MacGowan AP. Tigecycline pharmacokinetic/ pharmacodynamic update. J Antimicrob Chemother 2008;62 Suppl 1:111.

4. Jean SS, Hsieh TC, Hsu CW, et al. Comparison of the clinical efficacy between tigecycline plus extended-infusion imipenem and sulbactam plus imipenem against ventilatorassociated pneumonia with pneumonic extensively drugresistant Acinetobacter baumannii bacteremia, and correlation of clinical efficacy with in vitro synergy tests. J Microbiol Immunol Infect 2016;49:924-33.

5. Shen F, Han Q, Xie D, et al. Efficacy and safety of tigecycline for the treatment of severe infectious diseases: an updated meta-analysis of RCTs. Int J Infect Dis 2015;39:25-33.

6. Karaiskos I, Giamarellou H. Multidrug-resistant and extensively drug-resistant Gram-negative pathogens: current and emerging therapeutic approaches. Expert Opin Pharmacother 2014;15:1351-70.

7. Madadi-Goli N, Moniri R, Bagherijosheghani S, et al. Sensitivity of levofloxacin in combination with ampicillin-sulbactam and tigecycline against multidrug- resistant Acinetobacter baumannii. Iran J Microbiol 2017;9:19-25.

8. Fan XC, Wang XH, Zhang JS, et al. Detection of carbapenem gene in Acinetobacter baumannii from Eastern Heilongiiang province. Chin J Microecology 2015,27:651-3.

9. Turton JF, Ward ME, Woodford N, et al. The role of ISAba1 in expression of OXA carbapenemase genes in Acinetobacter baumannii. Fems Microbiology Letters 2006;258:72-7.

10. Park GC, Choi JA, Jang SJ, et al. In Vitro Interactions of Antibiotic Combinations of Colistin, Tigecycline, and Doripenem Against Extensively Drug-Resistant and Multidrug-Resistant Acinetobacter baumannii. Ann Lab Med 2016;36:124-30.

11. Kazi M, Drego L, Nikam C, et al. Molecular characterization of carbapenem-resistant Enterobacteriaceae at a tertiary care laboratory in Mumbai. Eur J Clin Microbiol Infect Dis 2015;34:467-72.

12. Doern CD. When Does 2 Plus 2 Equal 5? A Review of Antimicrobial Synergy Testing. J Clin Microbiol 2014;52:4124-8.

13. Dijkshoorn L, Nemec A, Seifert H. An increasing threat in hospitals: multidrug-resistant Acinetobacter baumannii. Nat Rev Microbiol 2007;5:939-51.

14. Kengkla K, Kongpakwattana K, Saokaew S, et al. Comparative efficacy and safety of treatment options for MDR and XDR Acinetobacter baumannii infections: a systematic review and network meta-analysis. J Antimicrob Chemother 2018;73:22-32.

15. Patsalos PN, Fröscher W, Pisani F, et al. The Importance of Drug Interactions in Epilepsy Therapy. Epilepsia 2002;43:365-85.

16. Cullivan S, Brady DM, O'Callaghan DS. New Delhi Metallo- $\beta$-Lactamase-Producing Carbapenem-Resistant Enterobacteriacae Isolated From Bronchial Washings. Ir Med J 2017;110:663.

17. Idelevich EA, Freeborn DA, Seifert H, et al. Comparison of tigecycline susceptibility testing methods for multidrugresistant, Acinetobacter baumannii. Diagn Microbiol Infect Dis 2018;91:360-2.

18. Stein C, Makarewicz O, Bohnert JA, et al. Three Dimensional Checkerboard Synergy Analysis of Colistin, Meropenem, Tigecycline against Multidrug-Resistant Clinical Klebsiella pneumonia Isolates. PLoS One 2015;10:e0126479.

19. Bauer G, Berens C, Projan SJ, et al. Comparison of tetracycline and tigecycline binding to ribosomes mapped 
by dimethylsulphate and drug-directed $\mathrm{Fe} 2+$ cleavage of 16S rRNA. J Antimicrob Chemother 2004;53:592.

20. Park GC, Choi JA, Jang SJ, et al. In Vitro Interactions of Antibiotic Combinations of Colistin, Tigecycline, and Doripenem Against Extensively Drug-Resistant and Multidrug-Resistant Acinetobacter baumannii. Ann Lab Med 2016;36:124-30.

21. Hagihara M, Housman ST, Nicolau DP, et al. In Vitro

Cite this article as: Li J, Fu Y, Zhang J, Wang Y, Zhao Y, Fan X, Yu L, Wang Y, Zhang X, Li C. Efficacy of tigecycline monotherapy versus combination therapy with other antimicrobials against carbapenem-resistant Acinetobacter baumannii sequence type 2 in Heilongjiang Province. Ann Palliat Med 2019;8(5):651-659. doi: 10.21037/apm.2019.11.06
Pharmacodynamics of Polymyxin B and Tigecycline Alone and in Combination against Carbapenem-Resistant Acinetobacter baumannii. Antimicrob Agents Chemother 2014;58:874-9.

22. Rao GG, Ly NS, Diep J, et al. Combinatorial pharmacodynamics of polymyxin B and tigecycline against heteroresistant Acinetobacter baumannii. Int J Antimicrob Agents 2016;48:331-6. 